\title{
A REVIEW IN SCRATCH DETERIORATION PATTERNS FOR POLYMER AND ITS COMPOSITES
}

\author{
Ammar Emad Al-kawaz \\ emadammar79@yahoo.com
}

University of Babylon / College of Material/Department Polymer and Petrochemical Industry

\begin{abstract}
Recently, scratch technique is widely used for characterizing the mechanical properties of a wide range of surfaces. In the engineering applications and in order to determine the relative hardness of materials, coatings and paints evaluations, and appraisal a variety of material damage countenance when subjected to asperity of the hard counterface, a scratch test was successfully used. In the current work, we have reviewed theoretical models that have been developed in the field of scratching and the scratch way that used for characterization polymeric surfaces. In order to design scratch-resistant polymers, we also discussed the various wear and scratch damage mechanisms for polymer and its composites. In addition, we discussed the influence of types and concentration of filler on the wear and scratch resistance. The aim of this work is to review the theoretical models that have been developed in the field of scratching and the scratch way that used for characterization polymeric surfaces.
\end{abstract}

Keywords :Scratch, damage feature, lubricating phases, Hybrid Composites

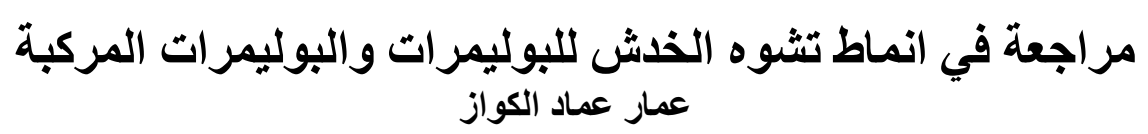

الخلاصة

في الآونة الأخيرة، نم استخدام تقنية الخدش على نطاق واسع لتوصيف الخصائص الميكانيكية لكثير من السطوح المختلفة.

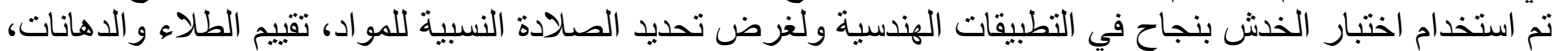

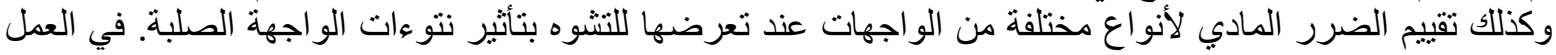

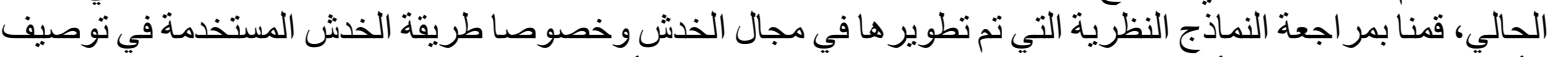

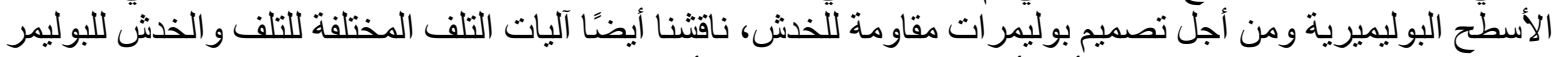

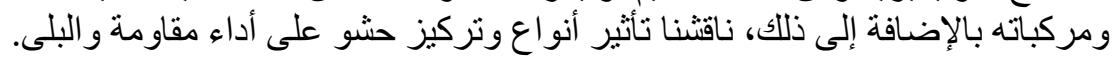




\section{INTRODUCTION}

Tribology is the science that includes a varied portion of machinery, surface engineering, friction, chemistry and lubrication between interacting similar and dissimilar surfaces in relative motion. The major target in a large portion of these cases is to diminish material harm or deterioration to a minimum or to increase their resistance (Dasari, Yu, and Mai 2009). Due to the importance of transparent polymers in many applications such as an optical lens and windows of aircraft, many research highlighted the weak characteristics of these materials such as poor abrasion resistance and weather ability (Lee and Jo 2002). The environmental temperature, moisture, the relative velocity of the interacting surfaces and the normal load, are a critical factors in the determined the surface properties of polymers. (Friedrich Klaus 2008). Most polymers in their pure state do not have acceptable wear resistance and perfect friction coefficient. The first solutions to solve this problem were using a metal coating layer deposit on the surface of polymer; the disadvantage of this manner is the huge difference in the "elastic strain" zones between the polymer substrate and coating layer (metal). While in the second generation, a high scratch resistance was obtained by increasing the hardness of the coating due to introduce polysiloxane and acrylic materials, which have elastic strain zone in the same limit with the substrate. Recently, nanomaterial was utilized as a filler to prepare anti-friction coating (Demirci, Gauthier, and Schirrer 2005)(Ammar Al-kawaz, Hadi, and Hamzah 2017). The use of fillers or slippage agents can improve the tribological properties of polymeric coatings that used in surface applications by reducing the friction coefficient and hence wear rate as well as to improve the mechanical properties such as elastic modulus, these fillers can increment the elastic response component in an elastoplastic behavior of the coating (A Al-kawaz et al. 2016) (Dasari, Yu, and Mai 2009) (Gauthier et al. 2006). A variety of fillers are utilized with polymer composites like inorganic particles to upgrade the mechanical properties of polymers and the stiffness, and toughness of the resultant composite is dependent on the particles size of the reinforcement particles(Friedrich, Zhang, and Schlarb 2005). Due to their low-cost and availability of nanoparticles $\mathrm{CaCO}_{3}$ are blended with PP in the nanometric scale in the polymer matrix to enhance mechanical and electrical properties, rheological and physical properties (Liang 2007)(Nano-cac, Behavior, and Properties 2006)(A. E. Al-kawaz 2018).

\section{FRICTION AND WEAR MECHANISMS OF POLYMER SURFACES}

Investigation how to determine the frictional force in a polymer surface during wear sliding against a harder counterpart is important to comprehend the overall tribological performance and the mechanism of tribological behavior of the polymeric surfaces(B J Briscoe 1981). If we impose that the counterface is hard adequately as a compare with polymer mate surface and suffer only moderate or no elastic damage, the frictional forces( tangential force)which represented as a result of the cohesive and interfacial work achieved on the polymer surface can be suggested by the two-term pattern (Brian J Briscoe and Sinha 2013a). In the frictional process, the included surface layer of the polymer can be categorized into two regions (Figure 1): the interfacial region with a depth of about $100 \mathrm{~nm}$ and according to the depth of the coating thickness, which called cohesive zone. Consequently, the resultant frictional force that generated from the "real contact area" of the interfacial region and the "shear stress" of the subsurface zone. Interfacial frictional work depends on many factors like the polymer hardness, glass transition temperature, crystallinity, counterface roughness, and this friction is result of the adhesive interactions between the polymer surfaces and the hard asperities (Friedrich Klaus 2008).

\section{POLYMER AND POLYMER COMPOSITES SCORCHING BEHAVIOR}

To study the material surface properties, a variety of techniques can be used such as indentation and scratch tests (H. Pelletier, Gauthier, and Schirrer 2009). Characterization 
tribological performance for polymers by scratching is the best replacement test to traditional wear, where a hard indenter is squeezed onto the material under normal load and moves approaching to the material, and the ability of the material to resist the mechanically induced surface damage under those conditions called scratch resistance. The apparent friction can be represented by the ratio of friction force to applied normal load on the tip that moved across the surface $\mu_{\text {app. }}$. Great friction can be realized by scratching process. In the front of the sample, the indenter was entirely resisted while in the rear half, only partially by elastically recovered material(B J Briscoe and Sinha 2003). During the scratching process, two main forces that act, the first force is a normal load which have vertically effect on the contact area between the indenter and the materials. while the frictional force which represents as a material's resistance against indenter dynamics in the tangential direction (Figure 2)(Mansha et al. 2011). The normal load can be defined as a scratch hardness and "tangential hardness" according to the "frictional force" and the "scratch hardness" can be calculated from these two forces (B J Briscoe and Sinha 2003). Thus, for elastic contact scratch hardness can be calculated from:

$$
H_{s}=\frac{4 W}{\pi(d)^{2}}
$$

Where $\mathrm{W}$ represents the applied load and $\mathrm{d}$ is the residual width of the groove after scratching experiment. Equation (1) applied when the material has a fully elastic behavior and completely recovered at the rear end of the movable indenter. While for the state of completely plastic or viscoelastic behavior, the scratched material at the contact will not completely support the rear half of indenter, so we can write: (B J Briscoe and Sinha 2003):

$H_{s}=\frac{8 w}{\pi d^{2}}$

Consequently, scratch hardness can be calculated from:

$H_{s}=\frac{q 4 W}{\pi d^{2}}$

Where q equal unity for material have a completely elastic behavior and 2 for material have a completely plastic behavior. While for material like polymers, the parameter $\mathrm{q}$ is between 1 and 2, due to viscoelastic and viscoplastic behavior, and the tangential hardness is generally particular as (B J Briscoe and Sinha 2003):

$H_{T}=\frac{F_{g}}{A_{T}}$

Where Fs represents the tangential force while $\mathrm{A}_{\mathrm{T}}$ is the contact area tangential direction.

It should be analysis the groove width and surface micro-features such as cracks, crazes, and plastic flows to illustrate the material damage from the scratch process. However, all polymers showed a complex scratch behavior and it is a function for the response of the material, test conditions like applied normal load and velocity, and, tip geometry(B. J. Briscoe 1998). Most of the polymers have viscoelastic and viscoplastic properties and characterization their scratch resistance is very complex and this because their behavior is strongly dependent conditions of the test such as contact geometry, normal load, loading rate, sliding velocity, surface topography (Hervé Pelletier, Mendibide, and Riche 2008). To enhance scratch performance for polymer a three suggested paths can be used: (Demirci, Gauthier, and Schirrer 2005)

$>$ Controlling the ratio between elastic modulus $E$ and ultimate yield stress $\sigma_{\text {yield }}$ in the range that makes the material having mechanical properties and acceptable scratch resistance.

$>$ Incorporating a strain-hardening effect in the bulk material, to raise the participation of the elastic in the elastic-plastic action.

$>$ Using coating film as anti-friction layer.

Depending on nature, volume fraction, size and geometry, matrix, and test conditions, nanoparticles play a major in controlling the resistance of polymers in the friction and wear. An efforts to study the tribological performance of PEEK and its nanocomposites showed that 
adding $\mathrm{Si} 3 \mathrm{~N} 4, \mathrm{SiO} 2$, and $\mathrm{SiC}$ in nanoscale have a great impact in the reduction of the friction of PEEK (Figure 3)(Brian J Briscoe and Sinha 2013b). So to recognize the impact of nanofiller, the paths of wear surfaces of pristine PEEK and $\mathrm{PEEK} / \mathrm{SiO}_{2}$ nanocomposite were investigated using SEM (Figure4). SEM images for the wear surfaces demonstrated a clear plowing behavior seem on the worn path of pure PEEK, while for the PEEK nanocomposite, the scuffing or plastic deformation was disappeared. This improvement in the tribological performance was attributed to forming a uniform and coherent transfer film on the counter surfaces in the case of nanofilled composite, while a dense, rough, and disconnected transfer layer was created in the case of pristine PEEK which caused a sharp plowing on the surface during scratching it by the hard counter surface. as a result, it could understand that the transfer films have a major function in enhancing the tribological performance of polymer nanocomposite, by modifying principle mechanisms of friction and wear(Friedrich Klaus 2008)(Brian J Briscoe and Sinha 2013b).

\section{THE MECHANISMS OF WEAR AND SCRATCH DEFORMATION FOR POLYMER AND ITS COMPOSITES}

During sliding two bodies against each other with an influence of a normal load, normally, the body having the softer surface loses its mechanical cohesion and created scrap from the contact region and consequentially wear deterioration polymers showing several styles of damage (ironing, ductile plowing, ductile/brittle machining, tearing, cracking, delamination, fatigue, abrasive, and adhesive) due to their strongly sensitive to scratch/wear effect. The indenter geometry, normal load, speed, and lubrication conditions are represent the main factors that effected scratch damage process (Hadal and Misra 2005)(Mansha et al. 2011).

In the case of polymer nanocomposites, the size, aspect ratio, hardness, concentration and particles orientation, the interfacial bond between polymer and particle, surface chemical reactions, and transfer films that may appear as a results of interaction the particles with counterface sliders, may an important role in controlling of the wear and scratch deformation , which make comprehension the tribological behavior of these materials is very complex(Friedrich Klaus 2008). In general, depending on the material properties and the state of applied load and quantity, the damage in polymers can be classified into two categories: ductile deterioration such as "shear yielding" and "ironing and brittle" deterioration which represented by crazing and cracking, for a variety of polymeric materials, it has been seen a variety scratch damage brows, like mar, fish-scale, parabolic crack, and material elimination(Britain 1998). Han Jiang et al (2009), they study the elastic, viscoelastic deformation and time dependency for four different types of polymers: strong ductile, weak ductile, weak brittle and strong brittle using scratch test under a low load and stress level. Plastic deformation takes place into the TPO substrate which figuration a cyclic concave deterioration in the direction of the scratching path as illustrated in figure(5a) shows the scratch deformation mechanism at the commuting zone from the primary deterioration to the fish-scale deterioration of TPO. With further increase in the normal load a fish scale damage style can be observed as illustrated in figure(5b). For PS the fake-fish-scale style is hardly apparent and conjugated cracks in micro-scale as can be illustrated by figure(5c)(Jiang, Browning, and Sue 2009). For PC and Epoxy, there is no evident scratch deterioration at a normal load below $70 \mathrm{~N}$. After that, the dominant deformation style is the parabolic cracks as can be seen from figure(6a), and because of the brittle nature, the cracks increased immediately in a brittle mode the moment that they are created and this "parabolic brittle" deterioration trait becomes further systematic and intensive with a growing scratch load(Hodge and Nieh 2004). For PC, the parabolic crack deterioration is a little unlike from those generated in friable matrices, due to the ductile nature of PC as can be shown in figure (6b). When the scratch load increases continuously, material removal will begin and a 
considerable material is dislocated from the surface. Altering the fish-scale pattern by the tear of this periodic fish-scale fashion of TPO was illustrated in figure(7a). Figure(7b) clarified the change from "pseudo-fish-scale" mode mingled with crazes/voids to the material dislocated of PS, while PC suffered a change state from "parabolic crack" mode to the dislocation mode as showed in figure(7c)(Jiang, Browning, and Sue 2009).

\section{THE IMPACT OF FILLERS TYPE ON THE WEAR PERFORMANCE OF POLYMER MATRICES}

The formulation of composites can be classified into two categories. First soft and lubricate phases are incorporated in a continuous relatively "hard phase' and the second" is a "rigid phase' reinforced 'soft phase' matrix (Stachowiak 2006). Utilizing the inorganic particles as reinforced filler have a great impact on enhancing the mechanical and tribological properties of polymers. A few factors employed to characterize the reinforcement performance of the composite matrix, such as microstructure of the resultant composite, size, and shape of the reinforcement agents, dispersibility and the interfacial bonding between filler and matrix. Used a low percentage from inorganic particles approximately $1-4$ vol. \% with a nanoscale is sufficient to improve the wear resistance of polymers (Wetzel et al. 2002).

\section{SOFT ADDITIVE IN A HARD MATRIX}

The major usefulness of introducing the soft phase in a hard matrix such a soft lubricating is to lower coefficient of friction and wear loss, by providing low shear stress or self-lubricant material in a hard matrix, such as adding graphite or PTFE to the PEEK to promote its tribological behavior. On the contrary, addition this material doesn't improve the mechanical performance like elastic modulus or tensile strength. The effect of adding a big amount of these soft phases can increase the wear rate due to the decadence in mechanical properties (elastic modulus and strength). $\mathrm{MoS}_{2}, \mathrm{CuS}$ and $\mathrm{CaF}_{2}$, aliphatic amides and silicone resins and fluids are the popular soft and lubricating material that can be incorporated in the polymer(Brian J Briscoe and Sinha 2013b)(Wypych 2005).

\section{HARD ADDITIVE IN A SOFT MATRIX}

In order to increment the matrix stiffness, a hard and strong phase should be incorporated with it. Regarding a friction, the self-lubricate nature of the matrix plays a major role, where the wear resistance is enhanced and that because of incorporating the hard phase that can enhance the ability to carry the load for the resultant composite. Many types of hard phases like glass, carbon fibers and many other types of hard particles like ceramic oxides are utilized with thermoplastic or thermoset matrices to produce polymer composites and showed variety effects. There are many kinds fillers such as glass fiber, carbon fiber, aramid fiber were widely used and have a great effect in raising the strength of the polymeric matrices like PTFE, polyamides, PEEK or phenolic resin. These composites mostly have a diminish wear rate compared with the pristine polymers, but still, number of factors like environment and fibres type have a great impact on the extent and the relative effectiveness of each filler (Friedrich Klaus 2008). Al-kawaz et al, they reinforced PMMA coating layer with MWCNTgrafted-PMMA and they showed that the coefficient of friction decreased with increasing the thickness of the PMMA layer on MWCNT walls or with an increasing weight fraction of polymer that grafted with the MWCNT surface (A Al-kawaz et al. 2016). Li-Jun Cui et al., they study the effect of using multi-wall carbon nanotubes (MWCNTs)functionalized with carboxyl groups and amino groups, on the friction and wear resistance of the epoxy resin utilizing M-2000 wear testing machine at a variety of sliding speeds and applied normal loads. The resultant composites which reinforced with MWCNTs showed a decrease in the 
friction coefficient and wear rate, and the later decreased with increment the loading of MWCNT(Cui et al. 2013)

\section{HYBRID POLYMER COMPOSITES}

Recently many efforts employed inorganic filler in nanoscale with traditional fillers to develop a new hybrid composite by combining (A. E. Al-kawaz 2018). Wetzel et al. (2002) they clarify that utilizing nano- $\mathrm{Al}_{2} \mathrm{O}_{3}(13 \mathrm{~nm})$ and micro-CaSiO${ }_{3}(4-15 \mu \mathrm{m})$ can enhance wear resistance and stiffness of epoxy(Wetzel et al. 2002). An acceptable level of friction and wear can be gained for a polymer matrix, due to the presence of both soft and hard strong phases and consequently, can promote the self-lubricating and load-carrying performance of the matrix. Merge of many types of hard filler such as glass fibre, carbon fiber or aramid fiber with soft and lubricating materials like PTFE, $\mathrm{MoS}_{2}$ or $\mathrm{CuS}_{2}$ could give the resultant composite superior wear resistance and minimum friction coefficient (Stachowiak 2006).

Guillaume Klein et al. They investigated the effect of adding SDS to the acryliccarboxylated latex and they found that increasing the percentage of SDS in the acrylic films caused increasing Young has and shear storage module, due to behave the aggregated crystallizes that forming in the bulk of the films, as a reinforcing filler. The migration of SDS to the surface can greatly decreasing shear stress due to the lubrication nature for SDS, which caused decreasing the friction coefficients(Houérou et al. 2017). Browning et al., they showed that a considerable increment in the scratch resistance can be gained by adding hybrid fillers talc and erucamide to TPO polymer, which revealed an acceptable development in the scratch performance have been accrued such as coefficient of friction and the resistance to plastic deformation, while there is no considerable alteration in scratch hardness. SEM images showed a clear accumulation in untreated talc particles which may prevent migrated erucamide to reached to the free surface of polymers and this explains the poor scratch resistance for the resultant composites (Browning et al. 2006). A considerable increase in the mechanical strength and acceptable bonding strength of the transfer film were performed due to the presence of nanoparticles. It is thus apparent that polymer reinforced with hybrid filler (inorganic nanoparticles and slipping agent) could provide synergy in terms development of wears performance.

\section{CONCLUSION}

Significant researches have been done in the area of tribological performance for thermoplastics and its nanocomposites which evidences that this subject is quite modern. For various kinds of polymers, different scratch deterioration styles have been specified, and with regard to the applied load level, the scratch deterioration styles, and the development process were described and discussed. Study the considerable factors that have, like the nature of the polymer, filler kind, filler concentration, volume, and form, as well as the response of the tribological behaviour of the polymer matrix by the test conditions.

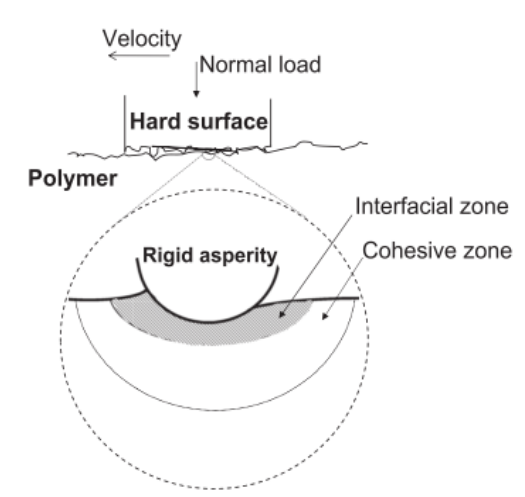

Fig.(1): The two represented expression pattern of friction and wear processes (Brian J Briscoe and

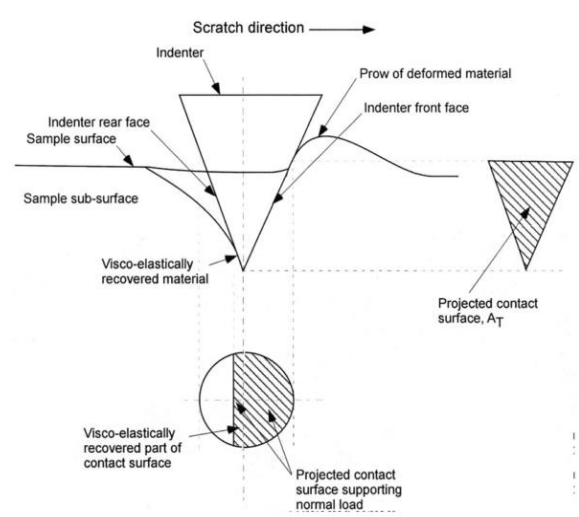

Fig.(2): Schematic the indenter during scratching process (B J Briscoe and Sinha 2003)(Brian J 
Sinha 2013a)

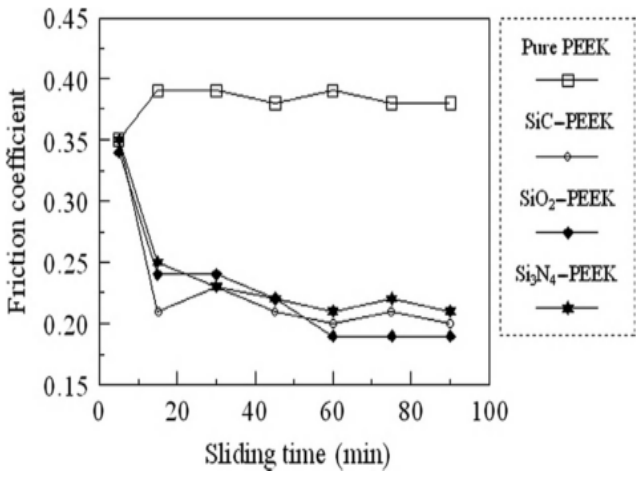

Briscoe and Sinha 2013b)

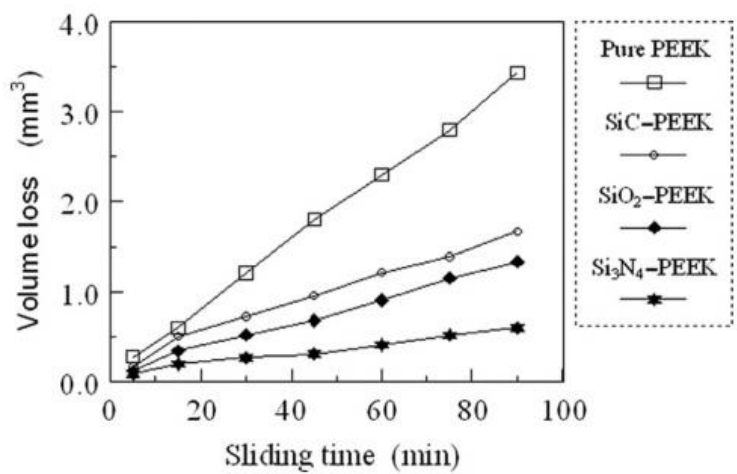

Fig.(3): Variation in a) friction coefficient and b) volume loss of PEEK and its composites using carbon steel ring (Brian J Briscoe and Sinha 2013b)
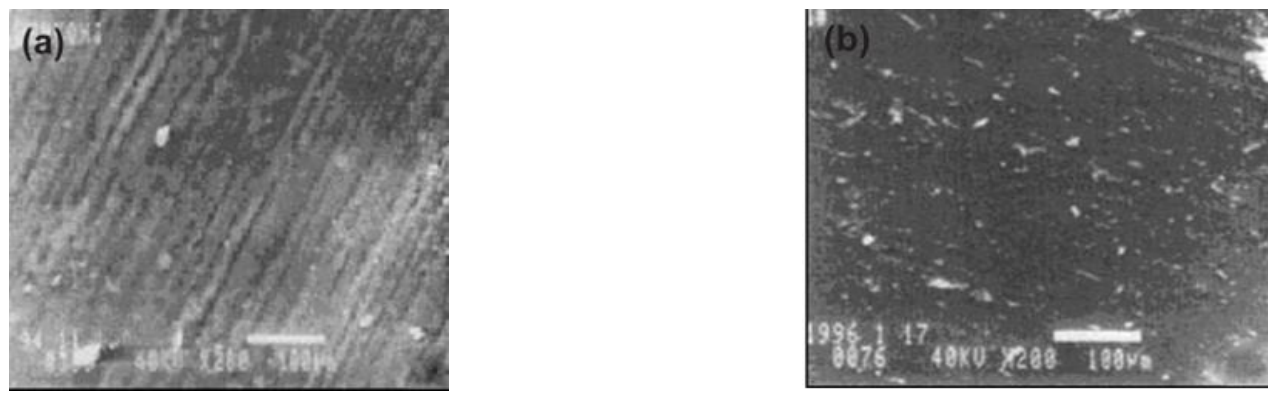

Fig.(4): SEM micrographs of the worn surfaces of the pure PEEK and its composites filled with 7.5 wt.\% nanometer $\mathrm{SiO}_{2}$ (load: $196 \mathrm{~N}$; sliding velocity: $0.445 \mathrm{~m} \mathrm{~s}^{-1}$; test duration: 90 min) (a) pure PEEK; (b) $\mathrm{SiO}_{2} / \mathrm{PEEK}($ Brian J Briscoe and Sinha 2013b)
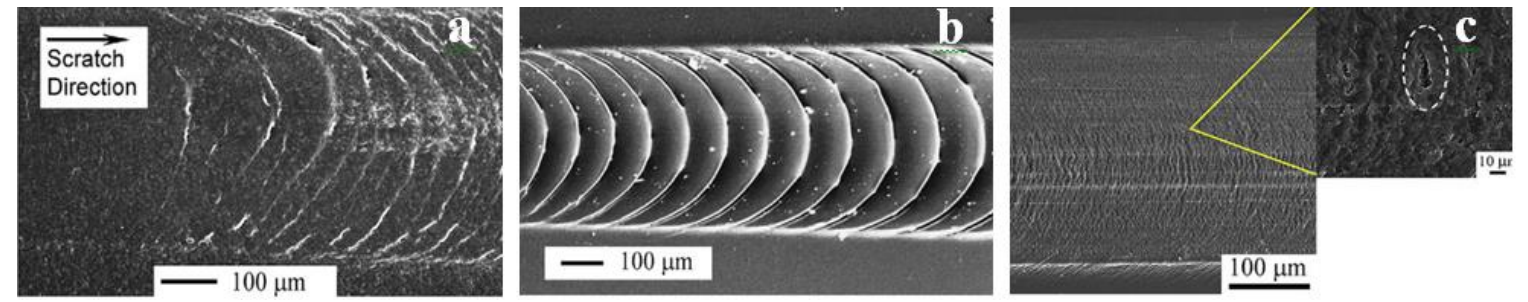

Fig.(5): SEM of "fish-scale" deterioration: (a)beginning of "fish-scale "genesis for TPO; (b) progressing "fish-scale" for TPO; and (c) "pseudo-fish-scale" style mixed with "crazes/ voids for PS" (Jiang, Browning, and Sue 2009)
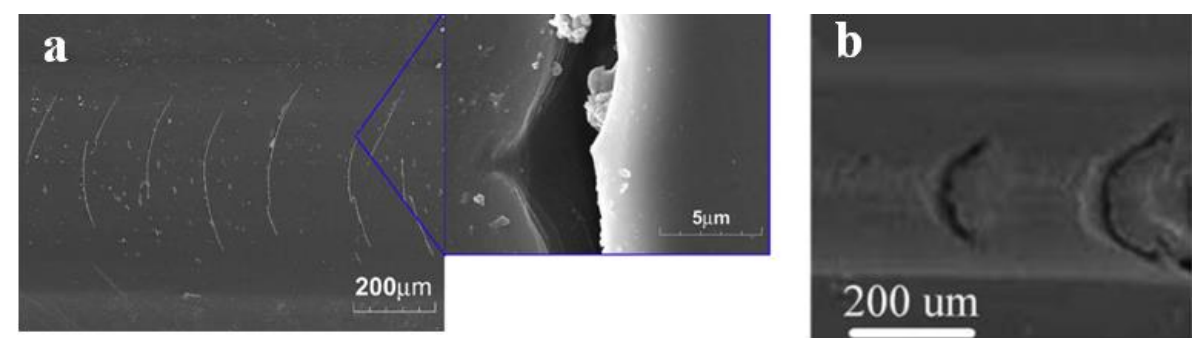

Fig.( 6): SEM of "parabolic crack" style in: (a) Epoxy; and (b) PC (Jiang, Browning, and Sue 2009) 

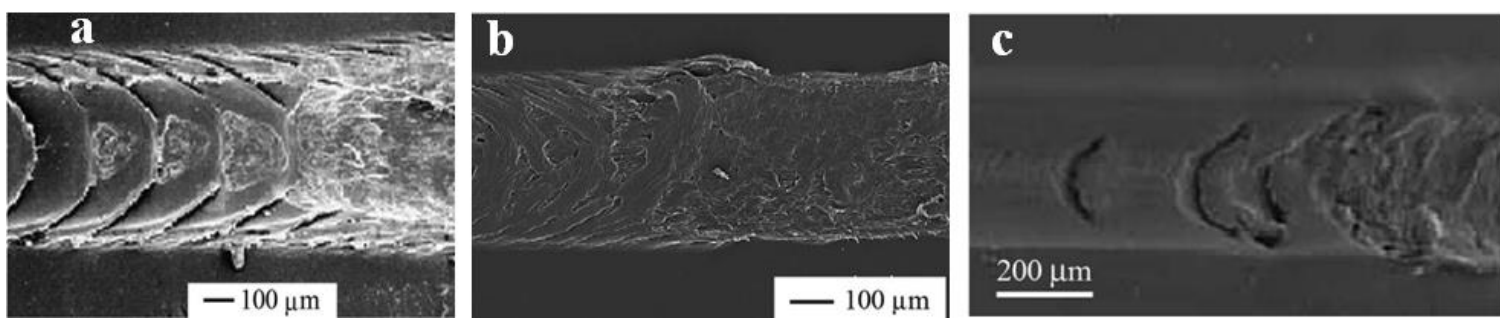

Fig.(7): SEM of the beginning material dislocation zone in (a) TPO; (b) PS; and (c) PC(Jiang, Browning, and Sue 2009)

\section{REFERENCES}

Al-kawaz, A, A Rubin, N Badi, C Blanck, L Jacomine, I Janowska, C Pham-huu, and C Gauthier. 2016. "Tribological and Mechanical Investigation of Acrylic-Based Nanocomposite Coatings Reinforced with PMMA-Grafted-MWCNT." Materials Chemistry and Physics. Elsevier B.V. https://doi.org/10.1016/j.matchemphys.2016.03.021.

Al-kawaz, Ammar Emad. 2018. "Recovery Behavior of Hardness Indentation of Epoxy Reinforced Hybrid Filler.” International Journal of Mechanical and Production Engineering Research and Development (IJMPERD) 8 (5): 375-82.

Al-kawaz, Ammar, Nizar Jawad Hadi, and Ahmed Fadhil Hamzah. 2017. "Study the Effect of Bi-Layers on the Friction and Impact Resistance of PMMA / Nano-Composite Hard Coatings" 12 (16): 6176-81.

Briscoe, B. J. 1998. "Isolated Contact Stress Deformations of Polymers: The Basis for Interpreting Polymer Tribology." Tribology International 31 (1-3): 121-26.

https://doi.org/10.1016/S0301-679X(98)00014-0.

Briscoe, B J. 1981. "Wear of Polymers: An Assay on Fundamental Aspects.” Tribology International 14 (August): 231-43.

Briscoe, B J, and S K Sinha. 2003. "Scratch Resistance and Localised Damage Characteristics of Polymer Surfaces - a Review," no. 10: 989-1002.

https://doi.org/10.1002/mawe.200300687.

Briscoe, Brian J, and Sujeet K Sinha. 2013a. 1.1 Introduction.

Friedrich Klaus 2013b" Tribological Applications of Polymers and Their Composites" Past, Present and Future Prospects. British Library Cataloguing in Publication Data.

Britain, Great. 1998. "Scratch Deformation of Methanol Plasticized Poly(methylmethacrylate) Surface" 39 (11): 2161-68.

Browning, Robert, Goy Teck Lim, Allan Moyse, Luyi Sun, and Hung-jue Sue. 2006. "Effects of Slip Agent and Talc Surface-Treatment on the Scratch Behavior of Thermoplastic Olefins." https://doi.org/10.1002/pen.

Cui, Li Jun, Hong Zhang Geng, Wen Yi Wang, Li Ting Chen, and Jing Gao. 2013.

"Functionalization of Multi-Wall Carbon Nanotubes to Reduce the Coefficient of the Friction and Improve the Wear Resistance of Multi-Wall Carbon Nanotube/epoxy Composites." Carbon 54 (August 2015): 277-82. https://doi.org/10.1016/j.carbon.2012.11.039. 
Dasari, Aravind, Zhong Zhen Yu, and Yiu Wing Mai. 2009. "Fundamental Aspects and Recent Progress on Wear/scratch Damage in Polymer Nanocomposites." Materials Science and Engineering R: Reports 63 (2): 31-80. https://doi.org/10.1016/j.mser.2008.10.001.

Demirci, I., C. Gauthier, and R. Schirrer. 2005. "Mechanical Analysis of the Damage of a Thin Polymeric Coating during Scratching: Role of the Ratio of the Coating Thickness to the Roughness of a Scratching Tip." Thin Solid Films 479 (1-2): 207-15. https://doi.org/10.1016/j.tsf.2004.11.194.

Friedrich, Klaus, Zhong Zhang, and Alois K. Schlarb. 2005. "Effects of Various Fillers on the Sliding Wear of Polymer Composites." Composites Science and Technology 65 (15-16 SPEC. ISS.): 2329-43. https://doi.org/10.1016/j.compscitech.2005.05.028.

Friedrich Klaus, Alois K. Schlarb. 2008. Tribology of Polymeric Nanocomposite. Edited by Alois K. Schlarb Friedrich Klaus. B.J. BRISC. Elsevier The Boulevard, Langford Lane, Kidlington, Oxford OX5 1GB, UK.

Gauthier, C., A. L. Durier, C. Fond, and R. Schirrer. 2006. "Scratching of a Coated Polymer and Mechanical Analysis of a Scratch Resistance Solution." Tribology International 39 (2): 88-98. https://doi.org/10.1016/j.triboint.2005.04.014.

Hadal, R S, and R D K Misra. 2005. "Scratch Deformation Behavior of Thermoplastic Materials with Significant Differences in Ductility" 398: 252-61. https://doi.org/10.1016/j.msea.2005.03.028.

Hodge, A M, and T G Nieh. 2004. "Evaluating Abrasive Wear of Amorphous Alloys Using Nanoscratch Technique" 12: 741-48. https://doi.org/10.1016/j.intermet.2004.02.014.

Houérou, Vincent Le, Christian Gauthier, Christian Gauthier, Yves Holl, Guillaume Klein, and Vincent Le Houe. 2017. "Friction Properties of Acrylic-Carboxylated Latex Films . 2 : Effect of Post Added Surfactant Effect of Post Added Surfactant." Tribiology International 57 (January). Elsevier: 257-65. https://doi.org/10.1016/j.triboint.2012.07.011.

Jiang, Han, Robert Browning, and Hung-jue Sue. 2009. "Understanding of Scratch-Induced Damage Mechanisms in Polymers.” Polymer 50 (16). Elsevier Ltd: 4056-65. https://doi.org/10.1016/j.polymer.2009.06.061.

Lee, Man Sung, and Nam Ju Jo. 2002. "Coating of Methyltriethoxysilane-Modified Colloidal Silica on Polymer Substrates for Abrasion Resistance." Journal of Sol-Gel Science and Technology 24 (2): 175-80. https://doi.org/10.1023/A:1015208328256.

Liang, J Z. 2007. "Evaluation of Dispersion of Nano- $\mathrm{CaCO}_{3}$ Particles in Polypropylene Matrix Based on Fractal Method" 38,1502-6. https://doi.org/10.1016/j.compositesa.2007.01.011.

Mansha, M, C Gauthier, P Gerard, and R Schirrer. 2011. "The Effect of Plasticization by Fatty Acid Amides on the Scratch Resistance of PMMA." Wear 271 (5-6). Elsevier B.V.: 671-79. https://doi.org/10.1016/j.wear.2010.12.089. 
Nano-cac, Oleic Acid-modified, Crystallization Behavior, and Mechanical Properties. 2006.

"Effect of Oleic Acid-Modified Nano-CaC03 on the Crystallization Behavior and Mechanical

S. Baoqing, L. Jipeng, W. Zhixue "Effect of Oleic Acid-Modified Nano-CaC03 on the Crystallization Behavior and Mechanical Properties of Polypropylene" Chinese J. Chem. Eng., 2006. 14 (6): 814-18.

Pelletier, H., C. Gauthier, and R. Schirrer. 2009. "Friction Effect on Contact Pressure during Indentation and Scratch into Amorphous Polymers." Materials Letters 63 (20). Elsevier B.V.: 1671-73. https://doi.org/10.1016/j.matlet.2009.05.015.

Pelletier, Hervé, Christophe Mendibide, and Antoine Riche. 2008. "Mechanical characterization of Polymeric Films Using Depth-Sensing Instrument: Correlation between Viscoelastic-Plastic Properties and Scratch Resistance." Progress in Organic Coatings 62 (2): 162-78. https://doi.org/10.1016/j.porgcoat.2007.10.009.

Stachowiak, Gwidon W., ed. 2006" Wear- Material Mechanisms and practice" British Library Cataloguing in Publication Data A.

Wetzel, Bernd, Frank Haupeiit, Klaus Friedrich, Ming Q I U Zhang, and M I N Z H I Rong. 2002. "Impact and Wear Resistance of Polymer Nanocomposites." Polymer Engineering \& Science 42 (9): 1919-27. https://doi.org/10.1002/pen.11084.

Wypych, George. 2005"Databook of Antiblocking, Release, and Slip Additives"ChemTec Publishing 38 Earswick Drive, Toronto, Ontario M1E 1C6, Canada. 